\title{
King's Fund forum consensus statement: screening for fetal and genetic abnormality
}

The fourth King's F und forum was held in London from 30 November to 2 December 1987. A panel of 12 listened to evidence from experts in public sessions attended by 250 people including professionals from many disciplines as well as the public and press. After closed sessions the panel discussed their report with the audience and the panel's consensus statement was then presented at a press conference.

Three factors have contributed to a growth in interest in the management of genetic and congenital impairments. Firstly, the continued decline in mortality and morbidity due to other causes has increased the proportion due to genetic and congenital abnormality and led to demand for improved management of these conditions. Secondly, the rapid advances in molecular biology have provided radically new means for identifying the carriers of deleterious genes. Thirdly, the Health for All initiative by the World Health Organisation has included a focus on disabled people.

Several concerns have been expressed about the development of screening programmes, particularly those in which termination of pregnancy is an option. There is fear that a de facto programme of crude eugenics might be introduced. The claims of the fetus and the principle of the sanctity of life have been urged as constraints on the mother's freedom of choice. There are concerns that the diversion of resources to screening may impair other services, including those for disabled people; that screening of high risk ethnic subgroups may foster racist attitudes; and that screening may lead to overmedicalisation of the process of childbearing. There has also been fear that there might be increased stigmatisation of disabled people and their families, particularly those who opt out of a screening programme.

A goal of our society is to promote the autonomy of its citizens, and health services should contribute towards this goal. Although economic considerations are proper determinants of choice between different ways of attaining a goal, economic arguments should not in themselves determine what goals are to be sought.

Screening is only one possible approach to reducing disability. The primary prevention of environmentally determined congenital impairments and improving the facilities and attitudes of society to physically or mentally impaired people must be components of a comprehensive approach. Screening should be seen as a means of acquiring information that increases the scope for choice by participants. Although selective termination of pregnancy is one option to which this may lead, the success of a screening programme should be judged not only by its effect on the prevalence of impairments at birth but by its total effect on the wellbeing of women and their families.

The panel comprised: J Grimley Evans (chairman), Eva Alberman, Ruth Ashton, Martin Bobrow, Peter Coe, Anthony Culyer, Marion Hall, Roger Higgs, Marianne Rigge, Hilary Rose, Alwyn Smith and Albert Weale.

Invited experts presenting evidence were: Ms E Anionwu, Professor D Brock, Dr H Cuckle, Professor G Dunstan, Professor M Ferguson Smith, Dr A Harding, Professor R Harris, Mr J Henderson, Professor B Hibbard, Mrs C Lavery, Dr S Macintyre, Ms M McTair, Dr B Modell, Dr M Pembrey, Dr M Richards, Professor C Rodeck, Mr S Thomas, Professor N Wald, and Professor Sir David Weatherall.
The purposes of screening for genetic and congenital disorders are as follows.

1. To assist in informed decision making before pregnancy. Accurate information on possible risks may allow some couples to avoid high risk pregnancies, while other couples may elect to embark on pregnancies that they would without this knowledge have avoided.

2. To provide the option of not continuing with an abnormal pregnancy or to enable the mother and her family to prepare for the care of a disabled child.

3. To allow optimal management of delivery and postnatal treatment when fetal abnormality has been identified.

Question 1: What kind of screening and diagnostic tests are possible for genetic and congenital disorders?

The disorders with which we are concerned include $(a)$ the "single gene" disorders, such as haemophilia, muscular dystrophy, and thalassaemia; (b) the chromosome disorders, such as Down's syndrome; and $(c)$ congenital malformations, such as neural tube defects.

There are two broad but overlapping categories of procedures: $(a)$ those that are cheap and safe and therefore suitable for screening total populations, and $(b)$ those that are expensive or invasive, or both, and suitable only for groups already known to be at high risk.

Multistage screening to define a population at high risk may begin simply by ascertaining age, family history, and ethnic origin-for example, cystic fibrosis is common in white populations, sickle cell disease in those of Afro-Caribbean ancestry, and Tay-Sachs disease in Ashkenazi Jews.

Tests for carrier state of inherited disorders, such as the haemoglobulinopathies and mucopolysaccharide disorders, can identify couples at high risk of having affected children. The techniques of the "new genetics" will soon include detection of the cystic fibrosis gene carried by about $5 \%$ of the population in the United Kingdom.

The level of risk at which a diagnostic test should be offered will depend on the natural course and severity of the condition screened for and the test's validity, safety, acceptability, availability, and cost. Gene markers for many of the common disorders, including haemophilia, sickle cell disease, muscular dystrophy, cystic fibrosis, and Huntington's chorea, are already available and have reduced the numbers of unaffected male fetuses being aborted for sex linked disorders.

Current techniques based largely on gene tracking need a detailed study of the family beforehand. More specific mutation site assays will circumvent this for many disorders but not necessarily those caused by a variety of mutations_-for example, Duchenne muscular dystrophy.

Testing for fetal chromosome abnormalities is commonly undertaken at fairly advanced maternal ages, as the birth prevalence of Down's syndrome is strongly age dependent. Recent evidence shows that a low maternal serum concentration of $\alpha$ fetoprotein at 16-20 weeks of pregnancy is an important independent predictor of Down's syndrome, which may increase the efficiency of detection of this disorder. 
Fetal material for laboratory analysis may be obtained by chorion villus sampling, by amniocentesis, or by sampling fetal blood or other tissues in the second trimester. Only chorion villus sampling is useful before the 16th week of gestation, but it is not applicable to the detection of neural tube defects. Neither the risks of chorion villus sampling nor the rates of error in subsequent chromosome analysis have yet been fully evaluated, but the procedure is thought to cause more miscarriages than amniocentesis.

Screening for neural tube defects is widely practised by measuring maternal serum $\alpha$ fetoprotein concentration at 16-20 weeks'gestation as estimated by ultrasonography. The diagnostic procedure may be an amniocentesis to obtain fluid for measurement of $\alpha$ fetoprotein concentration and acetylcholinesterase activity, with extremely high rates of accuracy (but with a small additional risk of miscarriage), or ultrasonic scanning, which is not invasive but seems to yield higher rates of false negative and false positive results. The widespread replacement of screening for $\alpha$ fetoprotein by ultrasound scanning would be premature until better data are available.

Routine ultrasound. scanning performed at early gestation for confirmation of gestational age and presence of a heart beat will sometimes incidentally detect fetal malformations. In contrast, detailed anomaly scanning at 18-20 weeks is, in experienced hands, highly effective in detecting many malformations.

Identification by ultrasonography of conditions such as diaphragmatic hernia or exomphalos in late pregnancy allows delivery at an appropriate time in a hospital with immediately available paediatric surgery.

Neonatal screening to detect treatable conditions (phenylketonuria, sickle cell diease, hypothyroidism) is widely practised, whereas screening for cystic fibrosis and muscular dystrophies has not come into common use.

\section{Question 2: What are the benefits and costs of these tests?}

There is evidence that some programmes pay for themselves from the resources saved by having fewer disabled people. If the condition is fairly common and causes serious disability these savings can be substantial. Even if this were not so such programmes might be justified by their social and clinical outcomes. At least 6000 (one in every 100) babies born alive each year in the United Kingdom are seriously impaired in spite of nearly 2000 planned terminations for fetal and genetic abnormalities.

In the past new procedures have not been subjected to scrutiny of cost and benefit, but evaluation research of this type is necessary given competing claims on resources. A characteristic of such research is that costs in the sense of value of resources used are generally presented as quantitative and monetary. The outcomes, whether positive or negative, are descriptive and qualitative and are often taken as no more than points for consideration. The principal justification for providing screening programmes lies in such currently unquantified effects. Examples of benefits are the provision of authoritative information, relief from uncertainty, support during a period of crisis, and the expansion of an individual's scope for exercising choice. Examples of potential harms are the introduction of worrying delays while confirmatory tests are conducted, the distress that may result from false positive results, and the illusory reassurance given by false negative results. Another set of considerations concerns long term social effects such as changes in the status and integration of disabled people.

If only monetary information is considered there is a danger that the quantified may drive out the important in a kind of Gresham's law of screening. This is a particular danger when the quantified costs of a service exceed the subsequent financial savings. A further difficulty occurs when the costs of the tests are borne by one sector of the community and the savings are found in another. This may happen when a preventive programme funded by the National Health Service reduces costs later for a family or a social services department- for example, for Down's syndrome.

The only secure way to avoid biased appraisals is to attempt to account comprehensively and imaginatively for all possible costs and benefits. The weights attached to the components may differ according to the level at which a decision is being made. Those used in determining a budget for a population would not necessarily correspond to those used in a clinical encounter.

\section{Question 3: What social and ethical issues arise?}

The development and improvement of screening services should not be seen as an alternative to improving services for people who have impairments. A woman's informed and considered decision not to participate in a screening programme should be respected and appropriate care and support offered to her, her baby, and her family. Decisions require the free and informed participation of the woman: when there is a conflict of interest between parents it must be the woman who ultimately decides. She should, however, be entitled to involve her partner as much as she wishes, in particular to support her during the course of a termination. If it is desirable to include relatives and partners in screening this must also be based on informed consent.

A woman's access to a screening or diagnostic test should be independent of any decision she may make about the continuation of the pregnancy.

Genetic tests bring particular problems of confidentiality. Providers must take adequate steps to safeguard the interest of the screened person. The woman should have access to information about herself and the pregnancy. Some parents prefer not to be told the sex of the fetus, and this wish should be respected. When the sex is revealed that fact alone should not be the reason for termination.

The early stages of pregnancy are not the best time to inform and educate people about the types, extent, and purposes of screening. Education should start in schools: health, including basic genetics, should be in the core curriculum.

Government and health authorities have an ethical responsibility to ensure that screening services are provided equitably. The quality of and access to these services should meet the reasonable expectations of an informed public. Doctors and other professionals have a duty to provide services that are both technically competent and sensitive to the personal dilemmas that screening entails.

There is no consensus about the meaningfulness or extent of any "rights" of the early fetus. Some people have deeply held views against abortion, but although such a personal view should be respected, people should be allowed to follow their own conscience in this matter. There is evidence that a conscientious objection to abortion on the grounds of fetal abnormality is the view of only a minority in our society.

The rapid pace of technical advance will open the prospect of prenatal testing for anomalies of a wide range of severity. Society may justifiably place limits on the types of conditions for which to provide testing.

\section{Questions 4 and 5: What are the criteria for providing screening programmes and for their organisation and monitoring?}

Screening for fetal and genetic disorders can be carried out on the fetus, the newborn, or would be parents. A programme of screening should ensure that each screening test is offered at the optimal time.

Although there should be a nationally agreed policy for providing screening programmes, the pattern of screening required dictates a need for facilities to be organised at supraregional, regional, and district levels depending on the prevalence of the disease and the complexity of the investigational procedures.

One person should have overall managerial responsibility for the entire process from public information and primary ascertainment to care and support after delivery. This person would be responsible for quality assurance, coordinating the relevant professionals, and fostering support networks with self help groups in the community. Such people should be identified at regional and district levels. There is disturbing evidence of current inadequacy in communication among professionals and between professionals and users of the services.

Once decisions have been made about what screening procedures 
are to be offered it will be possible to decide which aspects of the screening programme require to be associated with a specialised clinical genetics service and which can satisfactorily be carried out by hospital and community obstetric services. Basic pregnancy screening is best carried out as part of normal antenatal care.

The confirmation of dates and the screening procedures should be carried out as early in pregnancy as is possible, and delay will affect outcome.

The next stage of the screening programme entails the further investigation of those in whom the screening tests give positive results. Further investigation may require referral to more specialised services. In a considerable proportion of cases the necessary investigations will not be completed by the middle of the second trimester. Current proposals to remove the availability of abortion above 18 weeks would severely restrict the potential benefit of screening programmes and are opposed by the panel. Any reduction in the availability of abortion couched in terms of weeks and not in terms of viability will not deal adequately with this issue.

At present the pregnancy screening techniques with general application are largely limited to those concerned with neural tube defects, Down's syndrome, and the haemoglobinopathies. In future screening for heterozygotes for the haemoglobinopathies and possibly for common recessively determined disorders such as cystic fibrosis will seek to identify carriers during their prepregnancy period, and this will require a different pattern of organisation.

Carrier testing for the heterozygote state for those genes for which particular ethnic groups are at high risk must be sensitively performed to avoid any suggestion of racism and must involve the full support and understanding of the individuals and community concerned.

A regional genetic service will require an effective database, including some form of genetic register and a DNA bank.

Another essential requirement of a genetic and screening service is the provision of counselling. Experienced specialist counsellors should form part of a genetics service, but training in counselling will also need to be more widely provided for health professionals in obstetric and community services. There should be specialised genetic counselling available to mothers at every stage of the screening programme. If a termination of pregnancy ensues the mother should have access to a bereavement counselling service, which should be available in every district to those who have undergone termination of pregnancy for whatever reason. An introduction to the appropriate support groups may be helpful for mothers with affected fetuses whether terminated or not.

At a national level there will be a need to promote genetic services, and initiatives from the royal colleges on postgraduate training for this specialty are to be commended. There is evidence that basic education in modern genetics is deficient in the curriculum of medical students and other health professionals, and this should be remedied in basic and postbasic training.

The health education authority should initiate a specific programme to raise general public understanding of advances in genetics and of the developing services associated with them.

Monitoring and evaluation of screening services need to be organised in relation to both process and outcome. Definition of target groups makes it possible to assess the extent to which members of some groups have been offered screening, have taken it up, have been found to be positive, and have taken up intervention options. Whenever possible confirmation of abnormality in terminated pregnancies should be sought. Simple systematic statistical monitoring along these lines is a logical extension of suitably devised recording procedures. Because of cross boundary flows for the services district based records systems are inadequate.

Outcome monitoring is essential despite the undoubted difficulties posed by the breadth of the objectives of screening. Monitoring of changes in birth prevalence of the disorders for which screening is carried out provides an assessment of only one legitimate objective. Facilitating access and choice in matters of reproduction are much more difficult to monitor, and this may require specific research.

Research and development are required not only for the technical advances of more effective screening but also for the identification and assessment of service innovation; especially in respect of the nature and adequacy of counselling services.

The complexity of these issues suggests a need for planning and coordination at national level with a remit to see that the good quality services at present deployed in some regions should be available throughout the country. Services should be able to respond appropriately to the opportunities to be expected from the new genetic technologies. These developments seem inevitably to require new moneys not provided by the National Health Service.
If health care staff receive a needlestick injury should the patient concerned be tested for the human immunodeficiency virus (HIV) as well as for hepatitis infection?

In the event of needlestick injury to health care workers it is helpful to obtain a blood sample for human immunodeficiency virus (HIV) and hepatitis B testing from the patient concerned. The information provided from these tests provides a logical basis for reassurance, counselling, medical follow up, and, if necessary, hepatitis B prophylaxis. Recent information sought by the British Medical Association from Mr Michael Sherrard QC and Mr Ian Gatt has clarified the medicolegal position on HIV testing. ${ }^{1}$ Medical practitioners are warned that, as the law stands at present, consent of the patient is essential and it will be unwise for practitioners to adopt a course-that is, testing without consent-which exposes them to the possibility of both criminal and civil proceedings. There may be exceptional circumstancesfor example, an unconscious, critically ill patient-where treatment (or testing) without consent may be justified in the interests of the patient. This is considered to be unlikely for HIV testing because of the length of time taken to obtain a result. In the case of needlestick injuries the taking of a blood sample and its subsequent testing for HIV are unconnected with the care of the patient and no case for direct benefit can be made.

Now that the legal position has been so clearly and firmly defined for HIV testing the question arises whether consent should also be sought for venepuncture and hepatitis $B$ antigen testing. The same principles must apply and the practioe of testing without consent which has been common in the past must surely stop.

If consent to testing cannot be obtained counselling and follow up of a needlestick recipient must be initiated without information of the patient's HIV and hepatitis B state. In this case if the risk of hepatitis B is considered to be significantly high prophylaxis with hepatitis B immunoglobulin and vaccine may be justified.-D J JEFFRIES, consultant virologist, London. 1 Sherrard M, Gatt I. Human immunodeficiency virus (HIV) antibody testing. Guidance from an
opinion provided for the British Medical Association. Br Med $\mathcal{F}$ 1987;295:9!1-2.

What value does dipyridamole have in prevention of myocardial infarct and strokes?

Dipyridamole inhibits platelet aggregation by mechanisms that are not completely understood. This effect is potentiated by aspirin. Used alone, dipyridamole has not been shown to be of any use in preventing myocardial infarction or stroke. A combination of dipyridamole and aspirin reduced the incidence of coronary death and non-fatal myocardial infarction in two studies but was not significantly superior to aspirin alone. Similarly, there seems to be no difference between the combination and aspirin alone in the prevention of stroke, though both are better than placebo.-LINDA BEELEY, consultant clinical pharmacologist, Birmingham.

de Gaetano G, Cerletti C, Dejana E, and Vermylen J. Current issues in thrombosis prevention with antiplatelet drugs. Drugs 1986;31:517-49.

Anonymous. Doubts about dipyridamole as an antithrombolic drug. Drug and Therapeutics Bulletin 1984;22:25-7. 